and RADFs (GFR MG: 4.4 fold, MG: 4.8 fold) to GFR MG was observed compared to MG.

Discussion According to present knowledge and in comparison to results obtained from RASFs, SScDFs do not show the ability to migrate from their application site to internal organs. Adhesive properties do not differ from healthy controls. Nevertheless, SScDFs are mediators of organ fibrosis, but it seems that there is no contribution of SScDFs to the spreading of the disease.

\section{A10.2 ANTI-AT1R AND ANTI-ETAR AUTOANTIBODIES IN PATHOGENESIS OF SYSTEMIS SCLEROSIS}

doi:10.1136/annrheumdis-2013-203224.2

${ }^{1}$ Angela Kill, ${ }^{2}$ Mike 0 Becker, ' Jeannine Guenther, ${ }^{3}$ Harald Heidecke, ${ }^{2}$ Duska Dragun, 'Gabriela Riemekasten. 'Charité University Hospital, German Rheumatology Research Center, a Leibniz Institute, Berlin, Germany; ${ }^{2}$ Charité University Hospital, Berlin, Germany; ${ }^{3}$ CellTrend GmbH, Luckenwalde, Germany

Background and Objectives SSc is a prototypic multiorgan disease characterised by vascular damage, autoimmunity and fibrosis with still unkown aetiology. Recently identified functional autoantibodies simultaneously targeting the angiotensin-II type- 1 receptor (AT1R-Abs) and the endothelin-1 receptor type A (ETAR-Abs) were linked to vascular and fibrotic complications in SSc. Presence of both autoantibodies moreover predicted mortality due to cardiopulmonary complications implicating their contribution in SSc pathogenesis. Here, autoantibody mediated effects on endothelial cell activation and their blockade by receptor inhibitors were studied.

Materials and Methods Human microdermal endothelial cells-1 (HMEC-1) and human dermal fibroblasts were treated with IgG from SSc patients containing anti-AT1R and anti-ETAR Abs (SScIgG) or with IgG from healthy donors (NC-IgG) as negative control. In parallel, SSc-IgG treated cells were incubated with AT1R-and ETAR- antagonists alone and in combination. Activation of endothelial cells was assessed by qRT-PCR and sandwich ELISA and of fibroblasts with immunocytochemistry.

Results Human endothelial cells showed increased expression and production of the pro-inflammatory chemokine interleukin-8 (IL-8) upon treatment with SSc-IgG positive for anti-AT1R and antiETAR Abs compared to control treatment with NC-IgG as analysed on mRNA and on protein levels. Furthermore, endothelial cells showed increased expression of the adhesion molecules vascular cell adhesion molecule-1 (VCAM-1) and intercellular adhesion molecule-1 (ICAM-1) with SSc-IgG versus NC-IgG treatment on mRNA levels. Expression of IL-8 and VCAM-1 was significantly decreased by receptor inhibition. Human dermal fibroblasts showed increased collagen 1 production upon treatment with SSc-IgG versus NC-IgG that was also reduced by receptor inhibition.

Conclusions Our data sugests a direct involvement of anti-AT1R and anti-ETAR Abs in endothelial cell and fibroblasts activation that is mediated by AT1R and ETAR activation. Increased expression of IL-8 and of VCAM-1 and ICAM-1 indicate a direct endothelial cell activation and inflammation. Increased collagen 1 production indicates fibroblast activation and pro-fibrotic events. Therefore, anti-AT1R and anti-ETAR Abs induce pro-inflammatory and profibrotic events and could be directly involved in the pathogenesis of SSc. In vivo experiments are underway to analyse anti-AT1R and anti-ETAR Abs mediated pathogenic events in SSc.

\section{A10.3 ARTHRITIS IN PATIENTS WITH IDIOPATHIC INFLAMMATORY MYOPATHIES}

doi:10.1136/annrheumdis-2013-203224.3

\footnotetext{
1,2Martin Klein, 1,2Heřman Mann, 'Petra Hánová, 1,2Lenka Pleštilová, 1,2Jakub Závada, 1,2 Jiři Vencovský. 'Institute of Rheumatology, Prague, Czech Republic; '2Department of rheumatology $1{ }^{\text {st }}$ Faculty of Medicine, Charles University, Prague, Czech Republic
}

Background and Objectives Arthritis in idiopathic inflammatory myopathies (IIM) is frequently observed in clinical practise, but, to our knowledge, there is lack of systemic reports of prevalence and/ or characteristics of arthritis in myositis patients. The aim of this study is to determine prevalence of arthritis in IIM patients; it's relation to the course of the muscle disease; characteristics of arthritis with respect to seriousness, distribution and extent as well as it's relation to autoantibody profiles and others disease's characteristics.

Materials and Methods In this cross-sectional study, clinical aspects of disease, history of arthritis and autoantibody profiles were obtained from 106 consecutive patients with definite diagnosis of IIM. In all of them the 68-joint index was investigated. In 55 IIM patients and in 60 control patients with rheumatoid arthritis (RA) German Ultrasound Score 7 (US-7) was performed.

Results Arthritis at any time of course of myositis occurred in 65 patients (61.3\%); 42 had arthritis at the beginning of myositis (in 22 patients before and in 16 together with onset of muscle weakness). 52 patients presented arthritis at clinical examination (25 poly-, 17 oligo-, and 10 with monoarthritis). Most frequently affected joints were wrists $(21.7 \%)$ and shoulders, metacarpophalangeal, and proximal interphalangeal joints (20.8\%). From 29 anti-Jo-1 positive patients 28 had arthritis and significant association between arthritis and anti-Jo-1 positivity was found ( $p<0.0001) .39$ out of 55 $(70.9 \%)$ patients had Gray-Scale (GS) synovitis on ultrasound; in 34 of them also Power-Doppler (PD) positivity was found. Only 4 patients had ultrasonographic tenosynovitis and 3 had bony erosions. From 60 RA patients 57 (95\%) had GS synovitis, which was $\mathrm{PD}$-active in 54 . Tenosynovitis was found in 25 patients. $25 \mathrm{RA}$ patients had one or more joint erosions. Mean US-7 score as well as scores of individual joints or modality subscores were significantly lower in IIM than in RA, but, when compared only those patients with positive findings, the differences were found in total US-7 score, and GS-synovitis and PD-tenosynovitis subscores, but not in PD-synovitis, GS-tenosynovitis as well as in scores of individual joints.

Conclusions Our data suggest that arthritis is common feature of myositis. It is often present at the beginning of muscular manifestation of disease, or it even precedes the onset of muscle weakness. Most common presentation is symmetrical, non erosive polyarthritis affecting particularly wrists, shoulders, metacarpophalangeal and proximal interphalangeal joints of the hands. We confirmed strong association of arthritis with anti-Jo-1 antibody. Ultrasound investigation of joint involvement in IIM shows less frequent involvement than in RA, but comparable activity of synovitis measured by PD in affected joints. IIMs have less erosions and tenosynovitis than RA.

\section{A10.4 AUTOMATED AND STANDARDISED ANALYSIS FOR HIGH DIMENSIONAL CYTOMETRIC DATA PROVIDES NEW OPTIONS FOR COMPLEX CELL-ASSOCIATED BIOMARKER SCREENING}

doi:10.1136/annrheumdis-2013-203224.4

'Till Sörensen, '2Ursula Schulte-Wrede, 'Silvia Pade, ${ }^{2}$ Heike Hirseland, 'Gerd Burmester, ${ }^{2}$ Andreas Radbruch, ${ }^{2}$ Andreas Grützkau, 'Thomas Häupl. 'Dept. of Rheumatology and Clinical Immunology, Charité University Medicine, Berlin; ${ }^{2}$ German Arthritis Research Center, Berlin

Background and Objectives Flow cytometry (FCM) is widely used in clinical research and offers rapid and quantitative characterisation at single cell level. Traditional analysis is a semiautomated, time-consuming process of gating and successive 2-D projections, influenced by investigator-specific settings. With an increasing number of parameters for multiplexing, the manual analysis step is most limiting and impedes high throughput analysis in FCM. 